See discussions, stats, and author profiles for this publication at: https://www.researchgate.net/publication/340162682

\title{
A Mini Review on Low Carbon Steel in Rapid Cooling Process
}

Article · September 2019

CITATIONS

0

2 authors, including:

Norazlianie Sazali

Universiti Malaysia Pahang

83 PUBLICATIONS 256 CITATIONS

SEE PROFILE

Some of the authors of this publication are also working on these related projects:
READS

2

Project Effect of Intermediate Layer on Gas Separation Performance of Disk Supported Carbon Membrane View project 


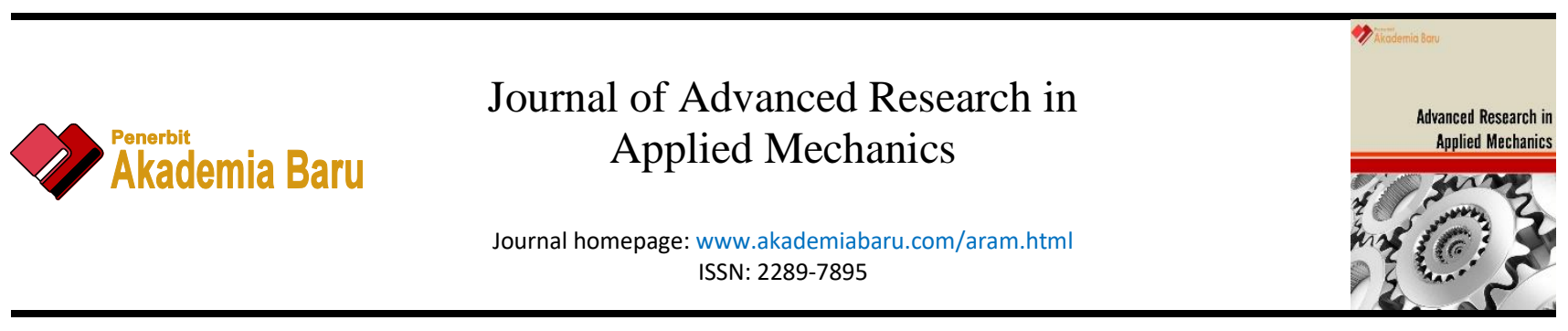

\title{
A Mini Review on Low Carbon Steel in Rapid Cooling Process
}

\author{
Siti Noradila Abdullah ${ }^{1}$, Norazlianie Sazali ${ }^{1,2, *}$ \\ 1 Structural Performance Material Engineering (SUPREME), Faculty of Mechanical \& Automotive Technology Engineering, Universiti Malaysia \\ Pahang, 26600 Pekan, Pahang, Malaysia \\ 2 Centre of Excellence for Advanced Research in Fluid Flow (CARIFF), Universiti Malaysia Pahang, Lebuhraya Tun Razak, 26300 Gambang, \\ Kuantan, Pahang, Malaysia
}

\section{ABSTRACT}

One of the latest technologies used in sheet metal production is hot stamping. It is a combination of forming and rapid cooling to create stronger components of sheet metal products. To handle a wide range of sheet metal that is suitable for hot forming, this short review emphasizes the study on microstructure changes during rapid cooling on different sheet metal thickness of low carbon steel. The mechanical properties like hardness, toughness and ductility can be altered by intense heat treating on steel and rapid cooling it to produce different mechanical properties. To change the characteristics of steels is by heat treating whereby altering the diffusion and cooling rate within its microstructure by changing the grain size at different phases and changing the molecular arrangement. The mechanical properties of the low carbon steel with different thickness may be different due to the microstructure behaviour after heat treating. Finally, the behaviour of the microstructure of low carbon steel changes with different thickness that is affecting the mechanical properties after heat treating and quenching process.

\section{Keywords:}

Low carbon steel; thickness; rapid cooling process; hardening; heat treatment

Copyright @ 2019 PENERBIT AKADEMIA BARU - All rights reserved

\section{Introduction}

This concise glossary of heat treatment terms has been embraced by the American Foundry Men's Association, the American Society for Metals, and the American Society for Testing and the Society of Automotive Engineers. To change the characteristics of metals and alloys is by heat treatment process whereby controlling the rate of dissemination and cooling within the microstructure to make them suitable for any kind of usage by changing the grain size at different phases and changing the molecular arrangement. For any process that engaged with changes of chemical properties and the physical is a heat treatment process by cooling or heating a metal. The technique for heat treatment includes, case hardening, annealing, tempering and precipitation strengthening, quenching and tempering. The mechanical properties itself can be modified such as hardness, strength, toughness, ductility and corrosion resistance. By intense heat treating on steel to produce different properties of hardness. This matters on the content of carbon in the steel like in Low carbon steel such as mild steel up to $0.4 \%$ carbon, in Medium carbon steel up to $0.8 \%$ carbon,

\footnotetext{
* Corresponding author.

E-mail address: azlianie@ump.edu.my (Norazlianie Sazali)
} 
and in High Carbon Steel up to $2 \%$ carbon. Low and medium carbon steel have insufficient amount of carbon to change their crystalline structure, as the results, the steel cannot be tempered or hardened. Medium carbon steel become harder regardless of whether it is hard and unfit to cut using a hacksaw. When steel is heated until it blazes with red color and is immersed in clean water immediately, the steel becomes very hard but also brittle. But, for easier to cut, or somewhat soft is the result for the blazing red-hot steel if is allowed to cool slowly.

The mechanical properties of any auxiliary metallic material with certain substance composition are elements of its miniaturized scale structure. The objective of heat treatment innovation is the improvement, or to control the mechanical properties by controlling the microstructure to coordinate the necessities constrained on a specific piece, or for a shaping procedure such as forming or machining innovation. The different mechanical properties of carbon steels will be resulted as different microstructures formed during cooling processes. Furthermore, the diffusionless transformations obtain the martensite formation which is the highest hardness in iron-carbon system and from diffusion transformation, lowest hardness is hardness is obtained which cause the ferrite or pearlite formation by a eutectoid reaction. Ferrite and pearlite obtained from austenite during slow cooling near the equilibrium while martensite is obtained during rapid cooling. Therefore, both steel mechanical properties and microstructure are related to steel thermal history.

Low carbon steel is one of the types of carbon steel which has low carbon content itself. As this type of materials has been selected in this study is to examine the effect of rapid cooling process on low carbon steel and the mechanical properties after undergo heat treatment processes. Therefore, the present-day work was planned to study the relationship among the microstructure, heat treatment and mechanical properties for rational selection of manufacturing process, properties and application for certain function. Rapid cooling is essential during industrial production processes. A type of heat-treating process, where immerse a work piece in water, or any fluids to acquire certain material properties. This procedure generally to keep up mechanical properties with a crystalline structure and the phase distribution that would lose during slow cooling process. Low carbon steel has been selected as this material is a type metal which has a comparatively low amount carbon which has a carbon content of $0.05 \%$ up to $0.25 \%$ by weight. Low carbon steel can be classified as hypoeutectoid steel based on its carbon content. This steel also has other elements such as manganese, silicon and small amounts of phosphorous to shield the integrity of the structure of metal iron by avoiding dislocations within the iron crystals.

Steel with low carbon steel has same properties as iron, which is easily to be forms and soft. As the carbon goes about as a solidifying agent, the strength of the steel increase by the large increments with the level of carbon contain. This made the metal becomes difficult to weld and less ductile but harder and stronger. Since low carbon steel cannot be strengthened by heat treating which can be only accomplished through cold working. This steel is machine-able and weld-able due to its softer and the outstanding ductility. Low carbon steel is mostly widely used of all steel products because it is cheaper compare to other types of steel. It is desirables properties such as ductility, strength and affordable price range make low carbon steel more likeable by industries. To allows parts to be fabricated in an easily formable soft state, steel can be heat treated. The alloy is hardened to increase wear and impact resistance and its strength, if there is enough carbon steel present. 


\section{Table 1}

Application of Low Carbon Steel based on carbon content

\begin{tabular}{cc}
\hline $\begin{array}{c}\text { Carbon Percentage, } \\
\%\end{array}$ & Applications \\
\hline $0.08-0.15$ & $\begin{array}{c}\text { Presswork, wire and rods for nails, screws, } \\
\text { and concrete reinforcement bar } \\
\text { Case carburising, quality, steel plate and } \\
\text { sections for structural work }\end{array}$ \\
$0.15-0.30$ & \begin{tabular}{c} 
sond \\
\hline
\end{tabular}
\end{tabular}

With every different heat treatment process of steels, the microstructure of steel will change. The study of microstructure also known as metallography or microscopy. The microstructures are the presentation of the structural characteristics of steel under microscopic state. A variety of microstructures of carbon steels are known as ferrite, cementite, pearlite, martensite and austenite. Resulting in partial transformations and the reaction of metastable phases from most of the phase transformations of interest will involve deviations from equilibrium microstructures. Ferrite, cementite and austenite can exist in harmony at the eutectoid temperature while pearlite existed by the solid-state transformation. This three stages, cementite, pearlite and ferrite are in this manner with the foremost constituents of the microstructure of plain carbon steels, if they have been exposed to slow cooling rates and stay away from the arrangement of transient stages. In this way, it is pivotal to watch the development of these stages and the nucleation, and to decide the highlights which control their morphology [1].

\section{Heat Treatment}

Heat treatment is a cooling or heating of metals in order to alter their mechanical and physical properties without deforming their shape. The objectives are to develop the physical and structural properties for some certain usage and for upcoming work of the metal. Heat treatment could be used to alter microstructure and properties of steels such as improving formability and machining and a method to strengthen the materials. To achieve the desired result, heat treatment includes the use of heating or cooling of the metals usually done to a very high or low temperature. The results of cooling and heating method are different because they are varying in the temperature and cooling rates used.

Different heat treating tasks incorporate heating and cooling of metals, types of heat treatment for ferrous metals are annealing, tempering, solidifying and case hardening. Normally, to harden a ferrous metal is by heating the metal to the needed temperature and cooling it by quenching method. This procedure increases the hardness and strength of metal. For tempering process, Steel is tempered at temperature which typically much lower than hardening temperatures due to the brittleness to relive the internal stresses and reduce its brittleness after being hardened.

For annealing, it is to make the metals more ductile and to refine their grain structures to relieve its internal stress and make it softer [9]. An ideal heat treatment for parts which require wear resistance surface is case hardening. The regular case hardening procedures are carburizing and nitriding. The result of this process when the metal cooled down, the outer surface becomes hard and soft in the insides but produced a very tough metal. Heat treatment process is used for the manufacture of components and machinery part, auto parts, gears and construction parts. Heat treating is an energy concentrated process that is carried out in different furnaces. This process generally is carried out in pit pot type furnace that is equipped with temperature control, hydraulic lifting and can be electrically heated or gas fired. Since these operations include large amount of heat 
transfer it cost a lot of energy wastage. To provide great environment, shortening heat treatment cycles can do so and can benefits financial through every saving.

\section{Austenite to Pearlite Transformations}

Transformation of austenite to pearlite begins when the temperature of austenite falls below the lower critical temperature. The formation starts by the formation of cementite crystal nuclei of austenite grain boundaries. Carbon diffuses from the surrounding austenite to the cementite, and the growth of carbide begins. As carbon diffuses, the adjacent austenite is depleted in carbon and transform to ferrite. With the formation of ferrite, there is a rejection of carbon from the ferrite region as effective.

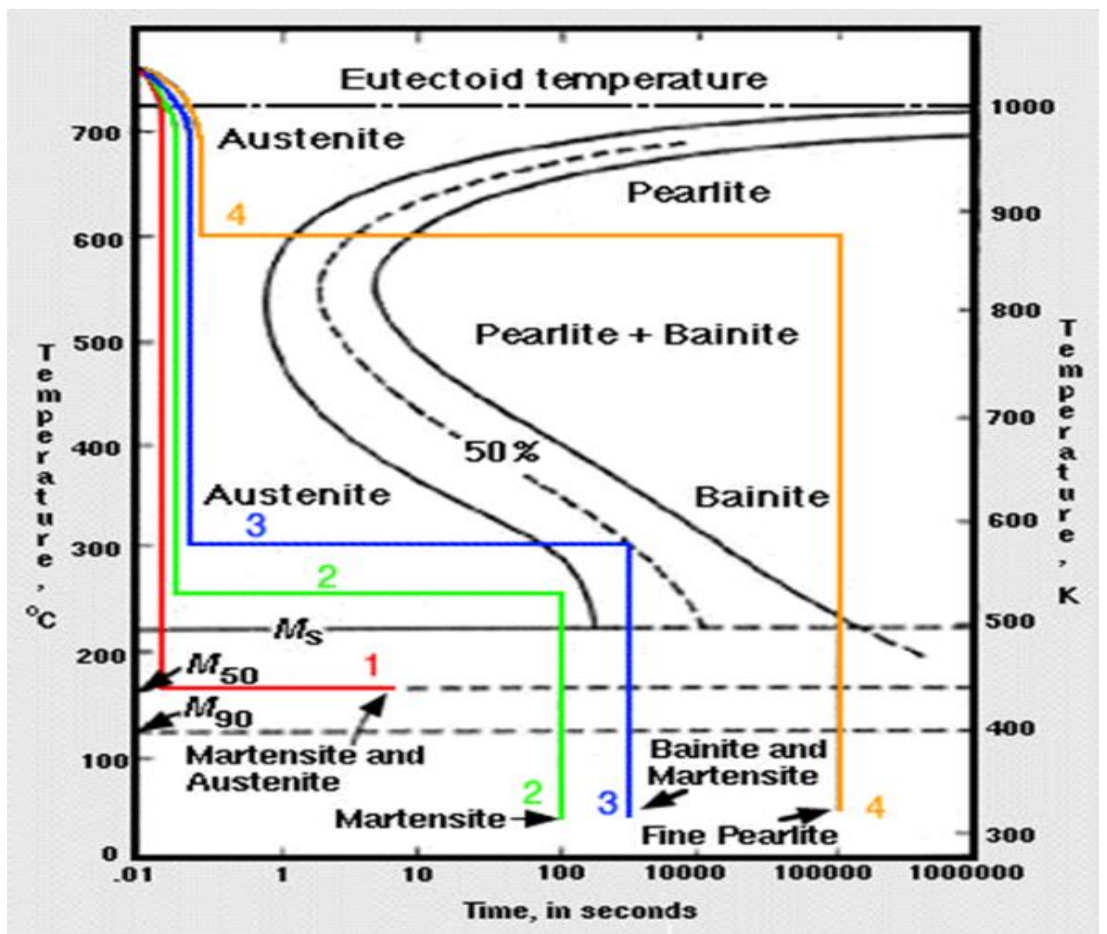

Fig. 1. Time-Temperature Transformation diagram of Carbon Steel

At the point when steel is heated up the critical temperature, austenite stage region is formed. This austenite phase in steel is stable only at lower critical temperature. At the point when the steel is chilled off at lower critical temperature, austenite will transform to another phases. The change of austenite is dependent with the temperature and time to transform. The relationship between these three variables shown in Time-Temperature Transformation diagram (TTT) in Figure 1. The TTT curve shows the transformation of austenite into different phases with temperature and time. The nose of the curve divides the curve into two parts. The upper part of the curve shows he transformation of austenite into pearlite phase. The down part of the curve, the transformation of austenite to bainite phase occurs between temperature of $550^{\circ} \mathrm{C}$ to $230^{\circ} \mathrm{C}$. During rapid cooling of steel to temperature below the nose of the curve, bainite is formed and is held isothermally until the transformation of bainite finished. Due to low main thrust or nucleation rate at close to eutectoid temperature, coarse pearlite are forms. Very fine pearlite is formed at the nose of TTT diagram and at lower temperature. Near the eutectoid temperature, the main thrust for the change is little on the grounds that the undercooling is low. The maximum rate is gotten at the nose of the curve as the undercooling 
increases change accelerates. The lowest part of the TTT curve shows the transformation of austenite to martensite. At a temperature of, the transformation of martensite from austenite is begins. As the temperature is decreasing, the martensitic formation will be increase below. To avoid nose of the curve to a temperature below, austenite must be cooled rapidly for the formation of martensite. The transformation of austenite to martensite is instantaneous. It is independent with time but dependent with temperature of transformation.

\section{Testing on Mechanical Properties}

\subsection{Rockwell Hardness Test}

Looking at different sorts of hardness testing methods, Rockwell hardness test is much simple procedure and give increasingly exact outcome results. This test method is utilized on all metals, except in condition where the product dent would be too large for the application where the test metal structure or surface conditions would present excess variation. Permanent depth of indentation created by a force on indenter can be measured by this test. First, a sample has applied with a force (preload/minor load) using a diamond or ball indenter. To lower the effects of surface finish, preload breaks through the surface. After holding the beginning test force for a predetermined stay time, the starting point depth of indentation is measured. After the preload, major load is applied to reach the total required test load which is an additional load. This force is held for measurement of time to consider elastic recovery. From that point onward, significant load is released, coming back to the beginning load. Subsequent to holding the earliest test force for a predefined stay time, the last depth imprint on the surface is measured. The difference in the baseline and final depth measurements is the Rockwell hardness value which has been derived. This distance between the baseline and final depth of indentation is changed to a hardness number. Lastly, the earliest test force is removed, and the indenter is removed from the test specimen. The recompense ought to be made for at least five indentation diameters between the center of the first indentation and the specimen edge or to the center of a second indentation where specimen thickness ought to be at any least fifteen times the indentation depth. Furthermore, the indentation on a smooth flat surface is depends on its accuracy.

Table 2

Hardness Testing Techniques [4]

\begin{tabular}{|c|c|c|c|c|}
\hline $\begin{array}{c}\text { Scale } \\
\text { symbol }\end{array}$ & Indenter & Total Load (kg) & Dial Numerals & Applications \\
\hline $\bar{A}$ & Diamond & 60 & Black & Cemented carbides, thin steel. \\
\hline B & $\begin{array}{c}1.588 \mathrm{~mm} \text { dia. Steel } \\
\text { ball }\end{array}$ & 100 & Red & Copper alloys, soft steels. \\
\hline $\mathrm{C}$ & Diamond & 150 & Black & Steel, hard cast iron. \\
\hline D & Diamond & 100 & Black & $\begin{array}{c}\text { Thin steel, medium depth case- } \\
\text { hardened steel }\end{array}$ \\
\hline $\mathrm{E}$ & $\begin{array}{c}3.175 \mathrm{~mm} \text { dia. Steel } \\
\text { ball }\end{array}$ & 100 & Red & $\begin{array}{c}\text { Cast iron, aluminium and } \\
\text { magnesium alloys. }\end{array}$ \\
\hline $\mathrm{F}$ & $\begin{array}{l}1.588 \mathrm{~mm} \text { dia. Steel } \\
\text { ball }\end{array}$ & 60 & Red & $\begin{array}{l}\text { Annealed copper alloys, thin soft } \\
\text { sheet metals. }\end{array}$ \\
\hline G & $\begin{array}{l}1.588 \mathrm{~mm} \text { dia. Steel } \\
\text { ball }\end{array}$ & 150 & Red & $\begin{array}{l}\text { Phosphor bronze, beryllium } \\
\text { copper, malleable iron. }\end{array}$ \\
\hline $\mathrm{H}$ & $\begin{array}{c}3.175 \mathrm{~mm} \text { dia. Steel } \\
\text { ball }\end{array}$ & 60 & Red & Aluminium, lead, zinc \\
\hline K & $\begin{array}{l}3.175 \mathrm{~mm} \text { dia. Steel } \\
\text { ball }\end{array}$ & 150 & Red & Soft bearing metals \\
\hline
\end{tabular}




\section{Heat Treatment Effects on Low Carbon Steel}

To give a good combination of toughness and strength from the martensitic phase, quenching and tempering heat treatments process have been used for a few decades applied to steels. The microstructure of low carbon steel includes of ferrite and small amount of pearlite. (Hasan MF., 2016) states in their research that the tensile strength of low carbon steel ASTM A-36 mild steel without any heat treated is $402.45 \mathrm{MPa}$ and the hardness is $69.8 \mathrm{BHN}$, the percentage elongation is $23.6 \%$ and reduction $56.24 \%$. The tensile strength and hardness of steel will rise due to mechanical properties while the ductility, elongation and reduction in area lessening. According to Hasan [5], normalizing does not soften the steel to the maximum and does not restore ductility much more done by annealing. The Brinell Hardness Number (BHN) of normalized is lesser than hardening but higher than annealing. Reheating the specimen to a certain temperature and cooling it at faster rate is a tempering process which reveal toughness at the cost of its hardness to an already hardened piece of steel. The decrease in tensile strength and hardness in the microstructure of the annealed sample by cooling can be linked with soft ferrite matrix. Due of the proper austenizing temperature and faster cooling rate, the increase in tensile strength and hardness compared to annealed and without heat treated. When low carbon steel was rapidly immersed from austenitic phase to room temperature, the austenite will transform into a mixture of martensite and finer pearlite. The hardness of steel increases both with cooling rate and the pearlite percentage. One of the reasons is due to the formation of martensite was the strengthening phases in steel. Due to the delay in the formation of pearlite and martensite at faster cooling rate, increasing the hardness value [5].

\section{Thermocouple}

A thermocouple is a connection between two conductors that switches a temperature difference into a potential difference through the thermoelectric effect. When two of these conductors is cooled or heated, a voltage is produced that can be connected back to the temperature. The thermocouple alloys are commonly used as wire. Thermocouple have different types with its own characteristics in terms of durability, temperature range, resistance to vibration and chemical and application compatibility. The most common thermocouple has been used are type J, K, T, \& E are "Base Metal" thermocouples, and type R, S, and B thermocouples are "Noble Metal" thermocouples, which are used in high temperature applications in industry. Thermocouples are globally used in industrial field because of them easily in hold or cold temperature and accurateness efficiency. Thermocouple useful for automatic measurements since it generates electric current which is much easier to get an electronic computer to measure a thermocouple temperature at regular intervals than to do it classically using a thermometer. Thermocouple is a very reasonable and have a high melting points which also durable to any different environment situation. Besides that, the maximum range of the thermocouple is limited by the diameter of the thermocouple wire where the calibration dictates the temperature range. So, a small diameter wire of thermocouple will not reach a full temperature range.

\section{Conclusions}

Transformation of austenite to martensite only takes place during higher cooling rates which is quenching process. The percentage of martensite forms on the austenite boundaries depends on the time taken the steel to cool. The faster the cooling rate of steel, the more percentage of martensite forms. Formation of pearlite rather than martensite occurs in air normalising process where the 
cooling rate is lower. Since the cooling rate is depending on the thickness of the steel, the thinner the steel, the faster it takes to cool into equilibrium temperature with high martensite or lower pearlite area formation. The microstructure development after heat treatment affects the mechanical properties of the steel. The hardness of the steel was mainly influenced by the grain development. However, the thickness also playing a main role in controlling the growth of the grain. Distribution of pearlite grain increase with the lower thickness of steel, whereas the hardness of the steel increase with the decreasing of its thickness. As the cooling rate of water quenching is higher than normalising, the hardness of water quenched was higher than normalising. On the other hand, the thinner the thickness of the steel, the more hardens the steel will be after rapid cooling process. It can be concluded that, the thickness of a low carbon steel can influence the microstructure growth and mechanical properties by using proper rapid cooling process.

\section{Acknowledgement}

Authors would like to extend their gratitude to Ministry of Higher Education Malaysia and Universiti Malaysia Pahang (UMP) with grant number RDU192315.

\section{References}

[1] Bhadeshia, H., \& Honeycombe, R. (2017). Formation of Martensite. Steels: Microstructure and Properties, 135177.

[2] Equbal, Md Israr, Parwez Alam, Rajkumar Ohdar, K. A. Anand, and M. S. Alam. "Effect of cooling rate on the microstructure and mechanical properties of medium carbon Steel." International Journal of Metallurgical Engineering 5, no. 2 (2016): 21-24.

[3] Salahudeen, Nurudeen, Abdulkarim S. Ahmed, H. Ala'a, Mohammed Dauda, Saidu M. Waziri, Baba Y. Jibril, and Jamal Al-Sabahi. "Synthesis, characterization and adsorption study of nano-sized activated alumina synthesized from kaolin using novel method." Powder Technology 280 (2015): 266-272.

[4] Callister, William D. Fundamentals of materials science and engineering. Vol. 471660817. London: Wiley, 2000.

[5] Hasan, M. F. "Analysis of mechanical behavior and microstructural characteristics change of ASTM A-36 steel applying various heat treatment." Journal of Material Science \& Engineering 5, no. 2 (2016): 1-6.

[6] Çalik, Adnan. "Effect of cooling rate on hardness and microstructure of AISI 1020, AISI 1040 and AISI 1060 Steels." International journal of Physical sciences 4, no. 9 (2009): 514-518.

[7] Ojha, Smriti, N. S. Mishra, and B. K. Jha. "Effect of cooling rate on the microstructure and mechanical properties of a C-Mn-Cr-B steel." Bulletin of Materials Science 38, no. 2 (2015): 531-536.

[8] Digges, Thomas G., Samuel J. Rosenberg, and Glenn W. Geil. Heat treatment and properties of iron and steel. No. NBS-MONO-88. NATIONAL BUREAU OF STANDARDS GAITHERSBURG MD, 1966.

[9] Adnan, Muhammad Adham. "Influence of Heat Treatment Process on Carbon Steel Pipe Corrosion in The Presence of Carbon Dioxide, CO2 and Acetic Acid, HAc." IRC, 2015.

[10] Hasan, M. F. "Analysis of mechanical behavior and microstructural characteristics change of ASTM A-36 steel applying various heat treatment." Journal of Material Science \& Engineering 5, no. 2 (2016): 1-6.

[11] Cerda, FM Castro, B. Schulz, D. Celentano, A. Monsalve, I. Sabirov, and R. H. Petrov. "Exploring the microstructure and tensile properties of cold-rolled low and medium carbon steels after ultrafast heating and quenching." Materials Science and Engineering: A 745 (2019): 509-516.

[12] Banadkouki, SS Ghasemi, and E. Fereiduni. "Effect of prior austenite carbon partitioning on martensite hardening variation in a low alloy ferrite-martensite dual phase steel." Materials Science and Engineering: A 619 (2014): 129136.

[13] Abbasi, Erfan, Quanshun Luo, and Dave Owens. "A comparison of microstructure and mechanical properties of lowalloy-medium-carbon steels after quench-hardening." Materials Science and Engineering: A 725 (2018): 65-75.

[14] Peng, Fei, Yunbo Xu, Jiayu Li, Xingli Gu, and Xu Wang. "Interaction of martensite and bainite transformations and its dependence on quenching temperature in intercritical quenching and partitioning steels." Materials \& Design 181 (2019): 107921.

[15] Ueda, Masaharu, and Kenji Matsuda. "Effects of carbon content and hardness on rolling contact fatigue resistance in heavily loaded pearlitic rail steels." Wear 444 (2020): 203120. 